\title{
Part B: The Feasibility and Acceptability of mI SMART, a Nurse-Led Technology Intervention for Multiple Chronic Conditions
}

\author{
Jennifer A. Mallow ${ }^{1}$, Laurie A. Theeke ${ }^{1}$, Rebecca Walls ${ }^{2}$, Elliott Theeke ${ }^{1}$, Brian K. Mallow ${ }^{3}$ \\ ${ }^{1}$ School of Nursing, West Virginia University, Morgantown, West Virginia, USA \\ ${ }^{2}$ Milan Puskar Health Right, Morgantown, West Virginia, USA \\ ${ }^{3}$ Sovern Run, LLC, Albright, West Virginia, USA \\ Email: "jamallow@hsc.wvu.edu, Itheeke@hsc.wvu.edu, etheeke@hsc.wvu.edu, bkmallow@gmail.com
}

Received 29 March 2016; accepted 25 April 2016; published 28 April 2016

Copyright @ 2016 by authors and Scientific Research Publishing Inc.

This work is licensed under the Creative Commons Attribution International License (CC BY).

http://creativecommons.org/licenses/by/4.0/

(c) (); Open Access

\section{Abstract}

Background: An opportunity to improve care of multiple chronic conditions for those living in rural areas of the country may exist through the use of technology. Integrating technology interventions into existing rural health systems allows for increased access to healthcare services and augments self-management ability for patients. However, questions remain about acceptability and feasibility of technology use in rural populations. The purpose of this paper is to present the feasibility of mI-SMART, a HIPAA compliant, web-based, structure of mHealth sensors and mobile devices designed to overcome the known health determinant of access to care for rural, chronically ill patients by using technology. Methods: The study was conducted at a primary-care clinic that provided healthcare at no or low cost to low income adults. Inclusion criteria encompassed adults, with at least one chronic condition, having at least $3^{\text {rd }}$ grade reading level, without having dementia/psychosis. Each participant was given a Nexus7 tablet and Bluetooth self-monitoring devices. Feasibility was evaluated in four ways and acceptability was evaluated with post-intervention questionnaires. Results: Thirty participants [mean age: 52 years (SD: 10.0, range: 29 - 74)] were majority female $(70 \%)$, white $(70 \%)$, married (60\%), high-school educated or less $(56.7 \%)$, impoverished (less than $\$ 20,000$ per annum (56.7\%), with multiple chronic conditions $(96.7 \%)$ ). During the trial, all participants were able to transmit data. No error messages were due to the mISMART system. Errors were user related and solved with technical support. Mean number of selfmonitor transmissions was 219.7 [(SD: 197.4), range: 1 - 733]. Participants logged into the system an average of 163. 1 [(SD: 169.7), range: 2 - 568] times and viewed an average of 1092.1 [(SD: 1205.6), range: 8 - 3851] intervention components. Over eighty-six percent of participants sent data for 12 weeks and $43.1 \%$ used the intervention for longer. Conclusions: The mI-SMART system is a feasible option for impoverished persons living in rural areas.

\footnotetext{
${ }^{*}$ Corresponding author.
}

How to cite this paper: Mallow, J.A., Theeke, L.A., Walls, R., Theeke, E. and Mallow, B.K. (2016) Part B: The Feasibility and Acceptability of mI SMART, a Nurse-Led Technology Intervention for Multiple Chronic Conditions. Open Journal of Nursing, 6, 323-332. http://dx.doi.org/10.4236/ojn.2016.64034 


\section{Keywords}

\section{Multiple Chronic Conditions, mHealth, Telehealth, Health Disparities, Nursing Informatics}

\section{Introduction}

An opportunity to improve care of multiple chronic conditions (MCC) for those living in rural areas of the country may exist through the use of technology. Integrating technology interventions into existing rural health systems may allow for increased access to healthcare services and augment self-management ability for patients [1] [2]. Examples of how technology has been used to improve care for chronic conditions includes: accessing information in electronic medical records, requesting medication refills and appointments through computerized systems, communicating using secure message systems, using connected devices to manage specific chronic conditions, using personal health records to track progress, interacting with on-line support groups, and using video conferencing to complete office visits [3]. The subsequent long-term effects of technology use lead to diminished health disparities, improved patient outcomes, and reduced healthcare costs [4].

Improving care for individuals with MCC is a priority in the United States. Approximately one in four Americans have two or more chronic conditions and individuals who experience MCC have an increased risk of dying, poor day-to-day functioning, and increased hospitalizations [5]. As such, the department of Health and Human Services developed a strategic framework to improve the health status of individuals with MCC [6]. There are four goals of the framework. First, the promotion of health system changes intended to improve the health of individuals with MCC. Second, use of self-care management and other services with empirical evidence of efficacy to improve care for individuals with MCC is supported. Next, providing better tools and information for those who deliver care to individuals with MCC is warranted. Lastly, facilitating research to fill knowledge gaps about interventions and systems for those with MCC is encouraged.

In order to address the strategic framework set forth by the department of human services, a new technology platform was developed. The platform, called mI SMART (Mobile Improvement of Self-Management Ability through Rural Technology), was developed using the model for developing complex nursing interventions [7]. The model suggests a progression for development of interventions with the purpose of increasing effectiveness, sustainability, and scalability. The theoretical underpinnings of mI SMART are based on the Chronic Care Model which consists of six interrelated system changes meant to make patient-centered, evidence based care easier to accomplish [8]. The major concepts in the Chronic Care Model are: health system, community support, selfmanagement support, decision support, clinical information systems, and delivery system design [9]. The Chronic Care Model is operationalized via mI SMART through a prepared healthcare team delivering planned interactions, self-management support with effective use of technology resources, integrated decision support, and supportive information technology (IT) which are designed to work together to strengthen the provider-patient relationship, improve communication, self-management ability, and improve health outcomes.

However, private industries have developed much of the available healthcare technology and technology is often being used in non-academic settings. Therefore, questions remain about acceptability and feasibility of technology use in rural populations. The purpose of this paper is to present the initial feasibility and acceptability of mI SMART, a nurse-led technology intervention for MCC in primary care.

\section{The mI SMART Platform}

The completed system combines a HIPAA compliant, web-based, structure of mHealth sensors and mobile devices to treat and monitor multiple chronic conditions. Different from what currently exists, mI SMART integrates primary care of multiple chronic conditions into one technology intervention. The mI SMART system allows patients to track diagnoses, medications, lab results, receive reminders for self-management, perform selfmonitoring, obtain feedback in real time, engage in education, and attend visits through video conferencing. The system displays a record database to patients and providers. Integration into existing Electronic Health Records is in progress. 


\section{Methods}

\subsection{Setting}

The study was conducted at Milan Puskar Health Right, a primary care clinic that provides health care at no or low cost to uninsured or underinsured, low income, adults aged 18 - 64 living in West Virginia. The clinic provides direct healthcare, health education, medications, and social services for this patient population. Our previous pilot studies using the EMR of the rural healthcare clinic where the intervention took place have identified that mean travel distance to this clinic for patients is 21 miles. The clinic has more than 28,000 patient encounters annually. The study was conducted between December 2, 2014 and December 8, 2015. This research study was reviewed by the West Virginia University Institutional Review Board (IRB) and was approved in accordance with 46 CFR 46.101b (Protocol \# 1501534474).

\subsection{Participants}

At-risk patients are those patients for whom attending frequent clinic visits is difficult due to a lack of transportation, working hours that are not conducive to regular office visits, or distance to the clinic is greater than the average distance. In addition, each participant must have a current diagnosis of Multiple Chronic Conditions that could be addressed using the mI SMART system. For example, participants can live with any combination of depression, diabetes, obesity, hypertension, or hyperlipidemia. Given these parameters, the identification of 30 participants was done through the recommendations of nurse practitioners and physicians in the clinic. The sample size was determined by the cost of the equipment and the amount of funding received. Inclusion criteria include being an adult age 18 - 64 with a diagnosis of multiple chronic illnesses and receiving care at the free clinic. Study participants are of both genders. Exclusion will include participants who do not speak or read English at a $3^{\text {rd }}$ grade reading level, and those with dementia or psychosis that would prevent on-going education and communication.

\subsection{The Intervention}

The mI SMART platform was implemented for 12 weeks with each recruited patient. The period of twelve weeks was chosen to overcome the potential for Hawthorne effect, allowing participants to establish a routine of using mI SMART. After the potential participants were identified by nurse practitioners and physicians in the clinic, they were contacted by the front desk staff and invited to participate. Potential participants were scheduled to come into the clinic for intervention explanation and completion of informed study consent. Each consenting participant was given a Nexus 7 tablet, and Bluetooth enabled self-monitoring devices that were individualized to their specific chronic illness diagnoses. The available devices were a glucometer, blood pressure cuff and scale. Instruction on how to use the tablet, the mI SMART platform, the self-monitoring devices, and personalized expectations of how and when to use the self-monitors were given to each participant. The participant was given the opportunity to ask questions. In addition to a verbal and hands-on demonstration, participants were given written and recorded instructions regarding how to use the system. Each participant was also given contact information for study staff for technical assistance. The participants answered pre-intervention surveys using the tablet prior to leaving the initial visit. Once the participant returned home, they began their 12 week intervention, using mI SMART to access the clinic instead of in-person visits. During the 12 weeks, each participant received education videos and live video conferencing with a health educator via the mI SMART platform. The content of the videos and education were dependent on the unique combination of chronic conditions of the participant. Tailored education included blood glucose monitoring, medication, nutrition, exercise, foot care, heart disease, complications of chronic illnesses, behavior change and more topics based on participant need. Patients received automated reminders for using the self-monitoring devices and taking medications. All clinic healthcare visits were completed using the mI SMART developed video conferencing system and were scheduled per the wishes of the provider and patient. The healthcare provider performed an assessment of the health history, limited physical exam, medication adjustments, and appropriate referrals via the developed video teleconferencing system. In addition to the automated feedback provided by the mI SMART system, a Registered Nurse reviewed and provided feedback and appropriate referral for finger stick logs, blood pressure logs and weights via the developed secure messaging system. At the end of the 12 week period each participant was able to keep the equipment provided. 


\subsection{Demographics}

Demographics were collected so that descriptive reports of the sample can be reported. The following demographics were collected: age, gender, ethnicity, marital status, education, income, number of chronic illnesses, and number of people in household.

\subsection{Feasibility and Acceptability}

The use of the developed technology to deliver the intervention and to collect and store data was evaluated in four ways: (1) reviewing the presence or absence of data in the database from each participant, (2) assessing the electronic activity logs and error messages for each participant, (3) assessing the electronic activity logs and error messages for each provider (4) analyzing the electronic activity logs of each application for number of times each application was used as well as common errors. Lastly, we assessed the acceptability of the technology with post intervention electronic patient satisfaction questionnaires. The questionnaires were accessible to the participants within the mI SMART system on the provided tablet and released to them on the last day of the intervention. Reminders were sent to each participant through the notification system within mI SMART and via telephone call to complete the questionnaires.

\subsection{Patient-Provider Communication \& Satisfaction}

Participants were provided questionnaires to assess communication based on 5-point Likert scale (see Figure 1 for the questionnaire used). The ease and convenience of communication, promptness of replies, quality and amount of information, and quality of care were evaluated. In addition, participants' satisfaction with the overall system was assessed. All communication requiring interaction between the patient and health care provider was stored in an activity log. The activity log was analyzed for frequencies of all patient-provider communication.

\section{Results}

\subsection{Demographics}

Thirty participants were enrolled and consented to participate in the study. The mean age of participants was 52 years (SD: 10.0, range: 29 - 74). The remainder of the participant demographics can be found in Table 1.

\subsection{Feasibility}

During the trial, all participants were able to transmit data from their self-monitoring devices located in their home in rural areas of West Virginia to our centralized servers. No error messages were received that were due to the mI-SMART system. All errors were user related and solved with technical support.

The most common errors were due to dual connections, third party vendor issues, hardware failure, and user unfamiliarity. The first type of error was due to security practices. When the tablet was connected to both a wifi connection and the mobile data service, the video conferencing would fail to connect to the provider. This problem was solved by instructing participants to turn off the wifi connection on the tablets for the duration of the study. The second type of error was due to issues with connectivity of third-party self-monitoring devices. A transmission error would occur that would delay the readings from reaching the mI SMART database. This was not an error that could be controlled internally. While the connection issue was usually resolved by the thirdparty vendor within 30 minutes, the tablet would announce a loss in connection. If this occurred at night, the announcement was disruptive to the patient. This was solved by contacting the vendor and instructing the patient to turn the audio off on the tablet when not in use. In addition, patients reported that the battery life of the tablet was about 12 hours and the glucometer batteries needed to be replaced at least once during the 12 week intervention. This was solved by instructing the patient to turn the blue-tooth connection off when not in use and by providing the patient with more batteries for the glucometer. Three blood pressure cuffs were damaged and two tablets were broken (a dog ate one and a baby dropped the other), each piece of damaged equipment was replaced. The user error problems were mostly due to an unfamiliarity with the technology and solved with education provided by phone or secure messaging.

Mean number of self-monitor transmissions for each participant was 219.7 [(SD: 197.4), range: 1 - 733] readings. Participants logged into the system an average of 163. 1 [(SD: 169.7), range: 2 - 568] times and viewed an 
Table 1. Demographics.

\begin{tabular}{|c|c|c|c|}
\hline Demographic & & $\mathbf{N}$ & $\%$ \\
\hline \multicolumn{4}{|c|}{ Gender } \\
\hline Male & & 9 & 30 \\
\hline Female & & 21 & 70 \\
\hline \multicolumn{4}{|c|}{ Ethnicity } \\
\hline African American & & 2 & 6.7 \\
\hline Asian/Pacific Islander & & 1 & 3.3 \\
\hline Hispanic & & 3 & 10 \\
\hline Native American & & 1 & 3.3 \\
\hline White & & 21 & 70 \\
\hline Other & & 2 & 6.7 \\
\hline \multicolumn{4}{|c|}{ Marital status } \\
\hline Divorced & & 4 & 13.3 \\
\hline Married & & 18 & 60 \\
\hline Separated & & 3 & 10 \\
\hline Single & & 2 & 6.7 \\
\hline Widowed & & 3 & 10 \\
\hline \multicolumn{4}{|c|}{ Education } \\
\hline Less than high school & & 7 & 23.3 \\
\hline High school/GED & & 10 & 33.3 \\
\hline Some College & & 5 & 16.7 \\
\hline 2 year college degree & & 3 & 10 \\
\hline 4 year college degree & & 3 & 10 \\
\hline Master's degree & & 2 & 6.7 \\
\hline \multicolumn{4}{|c|}{ Income } \\
\hline Less than $\$ 20,000$ & & 17 & 56.7 \\
\hline$\$ 20,000$ - \$34,999 & & 8 & 26.7 \\
\hline$\$ 35,000$ - \$49,999 & & 3 & 10 \\
\hline$\$ 50,000$ - $\$ 74,999$ & & 1 & 3.3 \\
\hline More than $\$ 100,000$ & & 1 & 3.3 \\
\hline \multicolumn{4}{|c|}{ Household size } \\
\hline Lives alone & & 4 & 13.3 \\
\hline 2 people in the home & & 8 & 26.7 \\
\hline 3 people in the home & & 4 & 13.3 \\
\hline 4 people in the home & & 3 & 10 \\
\hline 5 people in the home & & 3 & 10 \\
\hline Did not answer & & 8 & 26.7 \\
\hline \multicolumn{4}{|c|}{ Number of chronic illnesses } \\
\hline 1 & & 3.3 & 3.3 \\
\hline 2 & & 3 & 10 \\
\hline 3 & & 4 & 13.3 \\
\hline 4 & & 8 & 26.7 \\
\hline 5 & & 3 & 10 \\
\hline 6 & & 6 & 20 \\
\hline 7 & & 2 & 6.7 \\
\hline 9 & & 1 & 3.3 \\
\hline 10 & & 1 & 3.3 \\
\hline 12 & & 1 & 3.3 \\
\hline
\end{tabular}


mI SMART Satisfaction Questionnaire

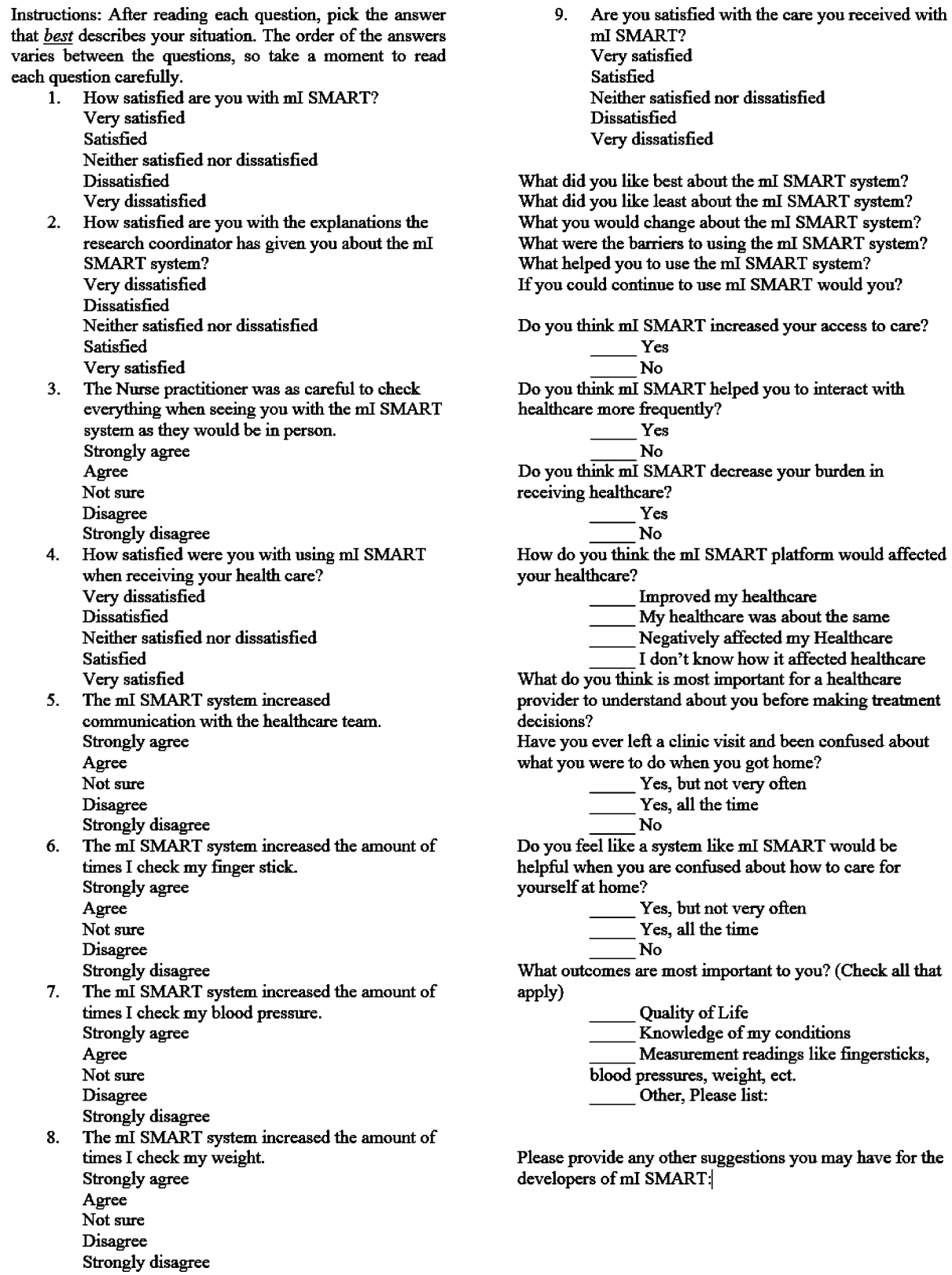

\section{Figure 1. Acceptability questionnaire.}

average of 1092.1 [(SD: 1205.6), range: 8 - 3851] intervention components (see Table 2 for details of intervention components viewed).

Over eighty-six percent of participants $(\mathrm{N}=26)$ sent data for the entire 12 weeks of the intervention and 46.61\% $(\mathrm{N}=14)$ used mI SMART for longer than the 12 week intervention. One participant began a new job that required travel and left the equipment at home. When he returned to his residence the intervention period was over. Two participants were lost to follow-up after the first day of monitoring. Follow-up with these participants was attempted but no response was received (see Figure 2. It is important to note that no-show rates for office visits in this particular clinic have been historically around $42 \%$ ). Hence, the $13.4 \%$ attrition rate in this study is promising. 


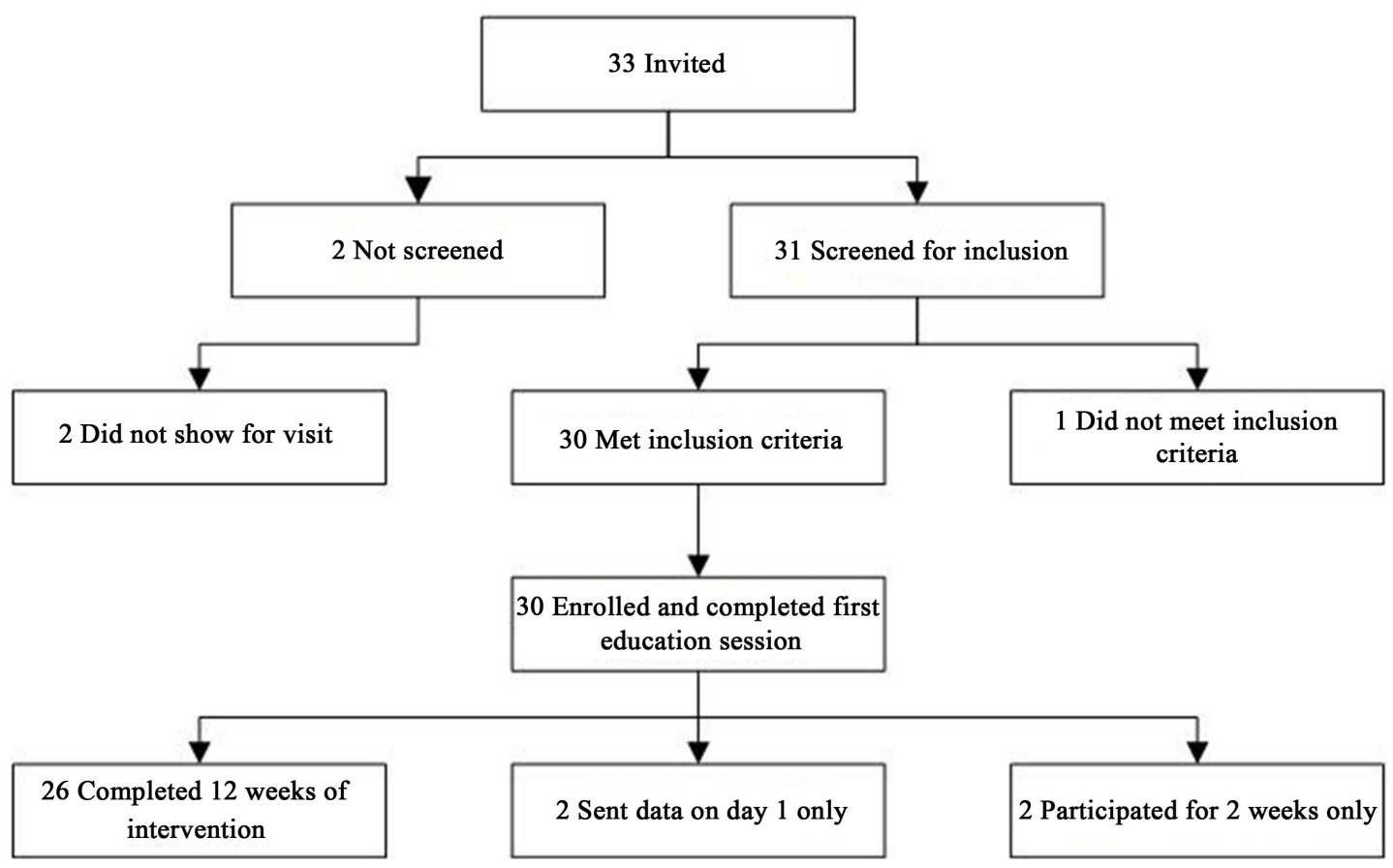

Figure 2. Summary of recruitment, enrollment, and completion by participants.

Table 2. Intervention component views.

\begin{tabular}{cccc}
\hline Intervention component & Range & SD & Mean \\
\hline Notifications/reminders & $1-2540$ & 821.5 & 542.20 \\
Self-monitor results & $1-362$ & 81.95 & 71.70 \\
Appointment list & $4-429$ & 84.10 & 69.33 \\
Messages & $1-250$ & 69.00 & 63.40 \\
Education & $1-41$ & 10.20 & 12.23 \\
Virtual visits (video conferencing) & $1-96$ & 18.31 & 10.77 \\
Prescription list & $1-50$ & 11.37 & 10.43 \\
Lab results & $1-103$ & 18.63 & 8.80 \\
Diagnosis list & $1-18$ & 3.85 & 4.23 \\
\hline
\end{tabular}

\subsection{Acceptability}

One-third of the participants $(\mathrm{N}=10)$ of the first trial of mI SMART responded to the post-intervention satisfaction questionnaire. Of the participants that responded, reported that they were either satisfied or very satisfied with the mI SMART platform. When asked if the healthcare providers were as careful when using mI SMART as they would be in person, nine respondents either agreed or strongly agreed and one participant was unsure. Eight participants felt that the mI SMART system increased communication with the healthcare team, 2 participants were not sure. Seven participants felt that using the system increased the amount of times that they performed self-monitoring such as taking glucometer, blood pressure and weight readings. Three participants reported that they completed their self-monitoring about the same amount using the mI SMART system as they would without. No one felt that the system decreased the amount of self-monitoring completed. Six participants answered the open-ended question, "What did you like best about the mi SMART system?” The responses included:

- "Increasing communication with my nurse", 
- "Easy to use",

- "Being more aware of my overall health",

- "Not having to go to the office",

- "Knowing that health professionals are looking over your shoulder all the time gives a very reassuring feeling”,

- "That my Doctor could monitor everything and that I could talk to her."

Five responses were received to the question "What did you like least about the mI SMART system?” two responses were related to the short battery life of the tablet, two responses were related to having unreliable internet connection in certain areas and one person stated "nothing”.

The changes participants would make to mI SMART included, two participants asking to "add a food log”, one asked for "better monitoring equipment" and one asked for "longer battery life of the tablet." There were two responses to the barriers of using the mI SMART system and they were both related to having to seek out the best place in their house to find internet connectivity. When asked what helped them to use the mI SMART system three respondents reported that the project coordinator was helpful, one said that they Health Right clinic was helpful, one reported all of the information they were given about the system, and one stated that being familiar with tablets and computers was helpful. Eight respondents reported that the system increased their access to care and that they interacted with healthcare providers more frequently. Eight participants reported that mI SMART improved their healthcare, one felt that the healthcare was about the same, and one didn't know how using the system affected their healthcare. When asked what outcomes were the most important to them, seven reported measures like glucose and weight, two reported quality of life, and one responded knowledge of conditions. All 10 respondents reported that if they could continue to use the mI SMART system they would.

\section{Discussion}

This study examined the feasibility and acceptability of mI SMART, a new technology intervention to improve access and outcomes for rural and underserved individuals living with multiple chronic conditions. West Virginia is the only state that is entirely located in the rural Appalachian mountain region. Prior to this trial, it was unknown if a technology platform using internet and wireless data was possible in such rural locations. It is noteworthy that all participants were able to transmit data from their homes at least once. Meaning, using internet interventions in rural populations is feasible. While no error messages were received in the database related to the mI SMART system, users did experience technical difficulties. Hence, patients having access to dedicated technical support will continue to be important as the intervention grows. Knowing the most common technical difficulties patients experience is important as future trials will include education on how to problem solve these issues. Hopefully, this will decrease frustration with the technology so that patients can focus on desired health outcomes.

Based on transmissions logs, most participants sent more than one self-monitoring reading per day for the duration of the intervention. Not surprisingly, the most used components of the intervention were interactive and required patients to engage with the system. Patient notifications and reminders was by far the most used part of the mI SMART intervention. However, the standard deviation was large and the range was wide. Hence, further investigation of which aspects of the notification system were desirable and effective is warranted. The least used components were education, prescriptions, laboratory results and diagnosis. To some degree, these results are understandable. If participants did not have recent laboratory tests or changes in diagnosis, it is expected that they would not interact with these components of the intervention. However, the lack of interaction with education components of the system warrants further investigation. The types of education, format of education, and amount of education patients' desire is still unknown.

Several factors affected patient acceptability of mI SMART. For example, one patient stopped using the system after 2 weeks, reporting that the frequent monitoring and small battery life of the tablet were frustrating and problematic. In addition, nearly half of the participants interacted with the system for longer than the 12 week intervention. The participants were only given 12 weeks of data service. Hence, this finding is interesting for two reasons. First, this would require participants to purchase their own data plans to continue to use the system, further supporting that internet interventions are feasible in rural and low resource environments. Secondly, the patients found the intervention acceptable enough to continue.

Despite reminders, the limited number of participants who completed acceptability surveys gave a limited 
view of the general acceptability of mI SMART. Though only 1/3 completed, one could logically infer from the continued use of mI SMART by the majority of participants, that it was useful. It is a key point that those who completed the surveys indicated that they would plan for continued use if available. More so, knowledge was gained about the similarities of mI SMART to in-person care which is similar to the literature. Most reported that they felt their care was similar to in-person care. Since mI SMART was noted to increase communication and self-monitoring, broader future evaluations of patient engagement in multiple self-care behaviors would be a strategic next step. Investigating other tablet options for longer battery life will be important in future trials.

\section{Limitations}

This study had several limitations. First, the study used a convenience sample of chronically ill adults receiving care at a free clinic. The sample was mostly female, white, married, with more than four chronic conditions living with low socioeconomic status. While the sample is representative of this particular state and clinic, the findings are not generalizable to the larger population. The low number of responses to post-intervention surveys limits what can be extrapolated related to acceptability of the intervention. Lastly, due to the small size of the clinic where the intervention was delivered, the feasibility and acceptability of the intervention for healthcare providers could not be evaluated. Hence, recommendations related to workflow, satisfaction of delivering care remotely, and cost of delivering the intervention cannot be assessed.

\section{Conclusion}

This study adds new knowledge about the feasibility and acceptability of mI SMART, a nurse led technology intervention for treating MCC in primary care. The development of the intervention is also available in part A of this publication series. In addition, the study team has completed the initial investigation of the effectiveness of mI SMART, which is reported in a separate manuscript. The mI SMART intervention has the potential to be a sustainable and scalable technology intervention to improve access to care after future longitudinal trails are accomplished. The next step will be to conduct larger longitudinal trials of mI SMART to appropriately assess the long-term health and health system benefits.

\section{Acknowledgements}

This project was supported by the Robert Wood Johnson Foundation Nurse Faculty Scholars Program. The Robert Wood Johnson Foundation Nurse Faculty Scholars Program is working to develop the next generation of national leaders in academic nursing through career development awards for outstanding junior nursing faculty. The program aims to strengthen the academic productivity and overall excellence of nursing schools by providing mentorship, leadership training and salary and research support to faculty.

\section{References}

[1] Khairat, S., Wijesinghe, N., Wolfson, J., Scott, R. and Simkus, R. (2014) Building a Multicenter Telehealth Network to Advance Chronic Disease Management. In: Mantas, J., Househ, M.S. and Hasman, A., Eds., Integrating Information Technology and Management for Quality of Care, Vol. 202, IOS Press, Amsterdam, 299-302.

[2] LeRoy, L., Bayliss, E., Domino, M., Miller, B.F., Rust, G., Gerteis, J., et al. (2014) The Agency for Healthcare Research and Quality Multiple Chronic Conditions Research Network: Overview of Research Contributions and Future Priorities. Medical Care, 52, S15-S22. http://dx.doi.org/10.1097/MLR.0000000000000095

[3] Mallow, J.A., Theeke, L.A., Barnes, E.R., Whetsel, T. and Mallow, B.K. (2014) Using mHealth Tools to Improve Rural Diabetes Care Guided by the Chronic Care Model. Online Journal of Rural Nursing and Health Care, 14, 43. http://dx.doi.org/10.14574/ojrnhc.v14i1.276

[4] Shi, L., Chen, C.-C., Nie, X., Zhu, J. and Hu, R. (2014) Racial and Socioeconomic Disparities in Access to Primary Care among People with Chronic Conditions. The Journal of the American Board of Family Medicine, 27, 189-198. http://dx.doi.org/10.3122/jabfm.2014.02.130246

[5] United States Department of Health \& Human Services (2015) HHS Initiative on Multiple Chronic Condition. 200 Independence Avenue, S.W.-Washington D.C. 20201: U.S. Department of Health \& Human Services. http://www.hhs.gov/ash/initiatives/mcc/\#_edn3

[6] United States Department of Health \& Human Services (2014) How Is HHS Addressing Multiple Chronic Conditions? 
200 Independence Avenue, S.W.-Washington D.C. 20201: U.S. Department of Health \& Human Services. http://www.hhs.gov/ash/initiatives/mcc/implementation/address-mcc.html\#framework

[7] Corry, M., Clarke, M., While, A.E. and Lalor, J. (2013) Developing Complex Interventions for Nursing: A Critical Review of Key Guidelines. Journal of Clinical Nursing, 22, 2366-2386. http://dx.doi.org/10.1111/jocn.12173

[8] Roger, V.L., Go, A.S., Lloyd-Jones, D.M., Benjamin, E.J., Berry, J.D., Borden, W.B., et al. (2012) Heart Disease and Stroke Statistics-2012 Update: A Report from the American Heart Association. Circulation, 125, e2-e220. http://dx.doi.org/10.1161/CIR.0b013e31823ac046

[9] Pullicino, P.M., McClure, L.A., Howard, V.J., Wadley, V.G., Safford, M.M., Meschia, J.F., et al. (2011) Identifying a High Stroke Risk Subgroup in Individuals with Heart Failure. Journal of Stroke and Cerebrovascular Diseases, 22, 620-626. 